\title{
Nikel-Kobalt (Ni-Co) Alaşımı Kaplamalarda Co Miktarının Mikroyapıya Etkisi
} The Effect of Co Content on The Microstructure of Nickel-Cobalt (Ni-Co) Alloy Coatings

\author{
Tuncay DİKİCi*1,2 \\ ${ }^{I}$ Dokuz Eylül Üniversitesi, Torbalı Meslek Yüksekokulu, Kaynak Teknolojisi Programı, 35860, İzmir \\ ${ }^{2}$ Dokuz Eylül Üniversitesi, Elektronik Malzemeler Üretim ve Uygulama Merkezi, 35390, İzmir
}

\begin{abstract}
• Geliş tarihi / Received: 06.06.2018 • Düzeltilerek geliş tarihi / Received in revised form: $31.07 .2018 \quad$ • Kabul tarihi / Accepted: 26.09 .2018
\end{abstract}
Öz

$\mathrm{Bu}$ çalışmada, Ni-Co alaşımlı kaplamalar elektrolitik yöntemle çelik malzeme yüzeyine sülfat banyosundan bir güç kaynağı yardımıyla hazırlanmıştır. Elektrolit içinde Co miktarındaki değişimin kaplamanın kompozisyon, faz yapısı ve morfoloji üzerine etkileri incelenmiştir. Elde edilen kaplamalar; X-ışını kırınım cihazı (XRD) ve taramalı elektron mikroskobu (SEM) ile karakterize edilmişlerdir. Sonuçlara göre, elektrolit içindeki farklı Co konsantrasyonu Ni-Co alaşımlı kaplama tabakasının özelliklerine etki etmektedir. Elektrolitteki Co oranının artışı ile birlikte alaşımlı kaplama tabakasında depozitlenen Co oranında ciddi bir artış söz konusudur. Ni-Co alaşımlı kaplama tabakasında YMK+HSP kristal yapısı artan Co miktarı ile HSP yapıya dönüşmektedir. Aynı zamanda alaşımlı kaplama tabakasındaki Co oranındaki artış ile birlikte yüzey morfolojisinde değişim gözlemlenmiştir ve daha granüler bir yapı oluşmuştur.

Anahtar kelimeler: Elektrolit, Ni-Co alaşımı, XRD, Yüzey morfolojisi

\begin{abstract}
In this study, Ni-Co alloy coatings were prepared on the steel substrates with electrodeposition method from a sulphate baths using power supply. The effect of Co content in electrolyte on the composition, phase structure and surface morphology of alloy coating were investigated. The prepared coatings were characterized using X-ray diffraction $(X R D)$ and scanning electron microscope (SEM). According to the results, Co concentration in the electrolyte affects the properties of the Ni-Co alloy coating layer. There is a serious increase of Co content deposited Ni-Co alloy coating when the Co concentration is increased in the electrolyte. The both FCC and HCP crystal structures of Ni-Co alloy coating layer began to transformin into HCP structure with increasing amount of Co content. At the same time, a change in the surface morphology was observed with an increase in Co ratio in the alloy coating layer and a more granular structure was formed.
\end{abstract}

Keywords: Electrolyte, Ni-Co alloy, XRD, Surface morphology

* Tuncay DIKİĊ; tuncay.dikici@deu.edu.tr; Tel: (0232) 8531820 (dâhili: 191); orcid.org/0000-0002-7004-9788 


\section{Giriş}

Son y1llarda, metal (Ni, Co ve $\mathrm{Fe}$ v.b.) ve alaşımlarının (Zn-Ni, Zn-Co, Zn-Fe v.b.) elektrokaplamasina olan ilgi sahip oldukları korozyon direnci, mekanik dayanım, manyetik ve termo-fiziksel özelliklerinden dolayı artmaktadır (Landolt, 2002; Oriňáková vd., 2006). Alaşımlı kaplamaların hazırlanmasındaki temel amaç sadece saf metal ile elde edilemeyen özelliklere bu sayede ulaşılmasıdır. Elektrokaplama tekniği ile üretilen nikel-kobalt (Ni-Co) gibi ikili alaşım kaplamaları; sahip oldukları korozyon direnci, yüksek mukavemet ve aşınma direnci gibi özelliklerinden dolayı havacilık, otomobil, bilgisayar sürücüleri ve diğer elektrik-elektronik endüstrisinde yaygın olarak kullanılmaktadır (Ishikawa vd., 1994; Qiao vd., 2006; Atuanya vd., 2017).

Saf metal ve alaşımların kaplanmasında elektrokaplama yöntemi; ekonomik, kolay, güvenilir ve tekrar edilebilir bir tekniktir (Yang ve Deng, 2011). Elektrolitik kaplama tekniği ile elde edilen tabakaların sertlik ve mukavemeti konvensiyonel metalürjik prosesle hazırlananlardan daha iyidir (Oriňáková vd., 2006). Ni-Co alaşımının elektro depositlenmesine ilişkin yürütülen çalışmalarda DC veya pulse akım kullanılmaktadır. Ni-Co alaşımının elektrokimyasal kaplaması kuraldışı davranış gösterebilir. Burada, daha soy metalden ziyade daha az soy metalin (Co) tercihli bir kaplanması söz konusudur. $\mathrm{Bu}$ durum, elektrolit içinden daha az soy metal iyonlarının $\left(\mathrm{Co}^{+2}\right)$ kaplama tabakasina daha yüksek oranda depozitlenmesi şeklinde gerçekleşir (Brenner, 1963; Zhuang, 2000).

Ni-Co alaşımlı kaplamaların kompozisyonu ve yüzey özellikleri büyük ölçüde çalışma parametreleri, elektrolit kompozisyonu, sicaklık, akım yoğunluğu ve $\mathrm{pH}$ gibi faktörlere bağlıdır (Lupi ve Pilone, 2001; Bai ve Hu, 2002; Gomez vd., 2006; Hassani vd., 2008). Bu alaşımlı kaplamaların özellikleri de büyük ölçüde Co miktarına bağlıdır. Proses parametrelerini değiştirerek Ni-Co alaşımlı kaplamaların yüzey morfolojisi ve mikroyapısı modifiye edilebilir. Kobalt içeriğine bağlı olarak, Ni-Co alaşımlı kaplamalar iki ana kategoriye ayrılır. Bunlar; (i) İyi koruyucu davranışa sahip (nikelce zengin alaşımlar) ve (ii) iyi manyetik özelliklere sahip (kobaltça zengin alaşımlar) alaşımlı kaplamalardır (Bakhit ve Akbari, 2013).

$\mathrm{Bu}$ çalışmanın amacı, elektrolit içerisindeki Co miktarındaki değişimin Ni-Co alaşımlı kaplama tabakasının yüzey özelliklerine olan etkilerinin incelenmesidir. Farklı Co oranı içeren elektrolitlerde gerçekleştirilen kaplama işlemleri sonucu Ni-Co alaşımına ait mikroyapı analizleri gerçekleştirilmiştir.

\section{Materyal ve Metot}

Kaplama altlığı olarak, $1 \mathrm{~mm}$ kalınlığında çelik sac numuneler kullanılmıştır. Deneysel çalışmada kullanilan bu sacin kimyasal kompozisyonuna dair bilgiler Tablo 1'de verilmiştir. Zımparalama sonrası altlık malzemenin yüzey pürüzlülüğ̈̈ değeri $\mathrm{R}_{\mathrm{a}}=0.32 \pm 0.05 \mu \mathrm{m}$ olarak ölçülmüştür. Örneklerin her biri yüzeyde muhtemel yağ, kir gibi kalıntıları temizlemek için alkali yağ alma işlemine tabi tutulmuştur. Sonrasında her bir örnek \%10'luk $\mathrm{HCl}$ içinde aktive edilip, bol su ile durulanmıştır.

Tablo1. Çelik sac levhanın kimyasal kompozisyonu

\begin{tabular}{llllll}
\hline Element & $\mathrm{C}$ & $\mathrm{P}$ & $\mathrm{S}$ & $\mathrm{Mn}$ & $\mathrm{Cu}$ \\
\hline \% ağırlık & 0.12 & 0.04 & 0.04 & 0.60 & 0.20 \\
\hline
\end{tabular}

$\mathrm{Bu}$ hazırlık aşamalarından sonra, yüzeyde bir oksit oluşumunu önlemek için örnekler hemen elektrolit içine yerleştirilmiştir. Kaplama banyo bileşenleri ve diğer çalışma parametrelerine ait bilgiler Tablo 2'de verilmiştir. Elektrolitik kaplama sistemine ait şematik gösterim Şekil 1'de mevcuttur. Kaplama işleminden sonra numuneler iyice yıkanmış ve kurutulmuştur.

Tablo 2. Kaplama banyosu bileşenleri ve çalışma parametreleri

\begin{tabular}{|c|c|c|c|c|c|c|}
\hline \multicolumn{5}{|c|}{ Elektrolit kompozisyonu } & \multicolumn{2}{|c|}{ Kaplama parametreleri } \\
\hline Kodlar & $\mathrm{NiSO}_{4} \cdot 6 \mathrm{H}_{2} \mathrm{O}$ & $\mathrm{CoSO}_{4} \cdot 7 \mathrm{H}_{2} \mathrm{O}$ & $\mathrm{H}_{3} \mathrm{BO}_{3}$ & $\mathrm{Na}_{2} \mathrm{SO}_{4}$ & Akım yoğunluğu & $3 \mathrm{~A} \cdot \mathrm{dm}^{-2}$ \\
\hline $\mathrm{NiCo}-5$ & $0.20 \mathrm{M}$ & $0.05 \mathrm{M}$ & $0.20 \mathrm{M}$ & $0.20 \mathrm{M}$ & Sicaklık & $24 \pm 0.5^{\circ} \mathrm{C}$ \\
\hline NiCo-10 & $0.20 \mathrm{M}$ & $0.10 \mathrm{M}$ & $0.20 \mathrm{M}$ & $0.20 \mathrm{M}$ & $\mathrm{pH}$ & 3 \\
\hline $\mathrm{NiCo}-20$ & $0.20 \mathrm{M}$ & $0.20 \mathrm{M}$ & $0.20 \mathrm{M}$ & $0.20 \mathrm{M}$ & Kaplama süresi & $10 \mathrm{dk}$. \\
\hline $\mathrm{NiCo}-40$ & $0.20 \mathrm{M}$ & $0.40 \mathrm{M}$ & $0.20 \mathrm{M}$ & $0.20 \mathrm{M}$ & Karıştırma hızı & $250 \mathrm{dv} / \mathrm{dk}$ \\
\hline
\end{tabular}




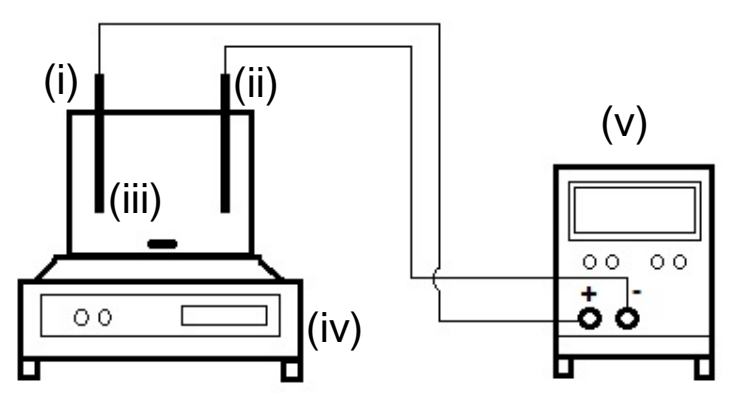

Şekil 1. Elektrolitik kaplama sistemi (i) anot (ii) katot (iii) elektrolit (iv) manyetik karıştırıcı ve (v) güç kaynağ 1

Hazırlanan Ni-Co alaşımlı kaplama tabakalarının faz yapılarının analizi, RIGAKU marka, D/MAX2200/PC model X-1şınları difraksiyonu (XRD, ARL X'TRA) cihazı ile gerçekleştirilmiştir. Kaplamaların mikroyapı ve yüzey morfolojisi JEOL JSM-6060 marka taramalı elektron mikroskobu (SEM) ile analiz edilmiştir. Taramalı elektron mikroskobuna bağlı enerji dağılım spektrometresi (EDS) cihazı ile alaşımlı kaplamalara yapılan nokta analizleri ile kimyasal bileşim yarı kantitatif olarak belirlenmiştir. Kaplama kalınlığı ölçümleri Elektro Physik eXacto marka cihazla gerçekleştirilmiştir.

\section{Bulgular ve Tartışma}

\section{1. $X R D$ analizi}

Farklı Co miktarı içeren elektrolit içinde $3 \mathrm{~A} \cdot \mathrm{dm}^{-2}$ akım yoğunluğunda gerçekleştirilen $\mathrm{Ni}$-Co alaşımlı kaplamaların faz kompozisyonu Şekil 2'de verilen XRD sonuçları kullanılarak incelenmiştir. Şekil 2'de görüldüğü üzere $2 \theta=42$, 44, 46 ve $77^{\circ}$ bulunan pikler Co elementine aittir. Genel olarak elde edilen XRD pikleri incelendiğinde Co miktarı arttıkça $2 \theta=42^{\circ}$ bulunan pikin şiddeti artmaktadır. Literatür bilgilerinde de mevcut olduğu gibi, Ni-Co alaşımında ağırlıkça \% Co miktarının kristal yapıyı etkilediği bilinmektedir.

Ağırlıkça \%49'dan daha düşük Co içeren nikelce zengin Ni-Co alaşımları YMK faz yapısına sahiptir (Golodnitsky vd., 2002). Co miktar1 ağırlıkça \%66' ya çıktığında ise (\%66-81) HSP yapı oluşmaya başlamakta ve Ni-Co alaşımının kristal yapis1 YMK+HSP kristalografik fazlarından oluşmaktadır. Co oran1 \%81'in üzerine çıkması durumunda ise HSP yapı daha dominant hale gelmektedir (Ma, vd., 2015). XRD sonucuna göre $2 \theta=42^{\circ}$ (100) doğrultusunda bulunan pikin baskın olması Ni-Co alaşımının
YMK ve HSP yapılardan oluştuğunu göstermektedir (Lupi vd., 2011). Elektrodepozit tekstürünün, büyüyen kristalografik düzlemlerin yüzey enerjisine bağlı olduğu yapılan çalışmalarla gösterilmiştir (Chen vd., 2006).

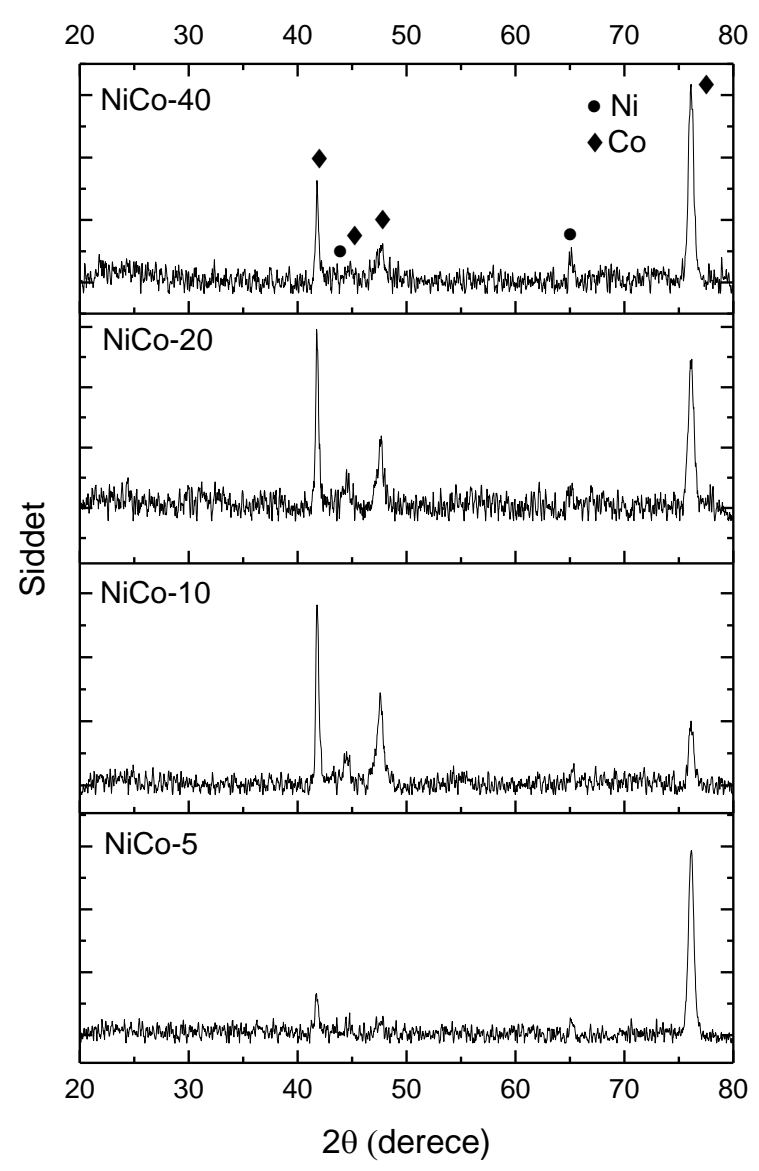

Şekil 2. XRD analizi

Şekil 3'de farklı kobalt konsantrasyonlarına sahip sülfat banyosunda hazırlanan Ni-Co alaşımlı kaplamalara ait yüzey morfolojileri verilmiştir. $\mathrm{Ni} / \mathrm{Co}=0.25$ olduğu elektrolit içinde hazırlanan kaplamada daha sıkı istifli kompakt bir yapının hakim olduğu ancak küçük partikül şeklindeki tanelerinde yap1 içinde yayıldığı bir morfoloji görülmektedir (Şekil 3a). Artan Co miktarı ile, granüler bir yapının oluştuğu ve sınırların belirginleştĭgi daha homojen bir yapının oluştuğu söylenebilir (Şekil 3b). $\quad \mathrm{Ni} / \mathrm{Co}=1.0 \quad$ olduğu elektrolit içinde yapılan kaplamada ise, tanelerin giderek büyüdüğü ve küçük çaptaki tanelerinde yoğun olduğu agrega görünümlü bir yüzey morfolojisi meydana gelmiştir (Şekil 3c). Co oranının en yüksek olduğu elektrolit içine yapılan kaplama sonucu, Ni-Co alaşımlı kaplama tabakasında ise agrega görünümlü tane çaplarının arttığı bir mikroyapı söz konusudur (Şekil 3d). 

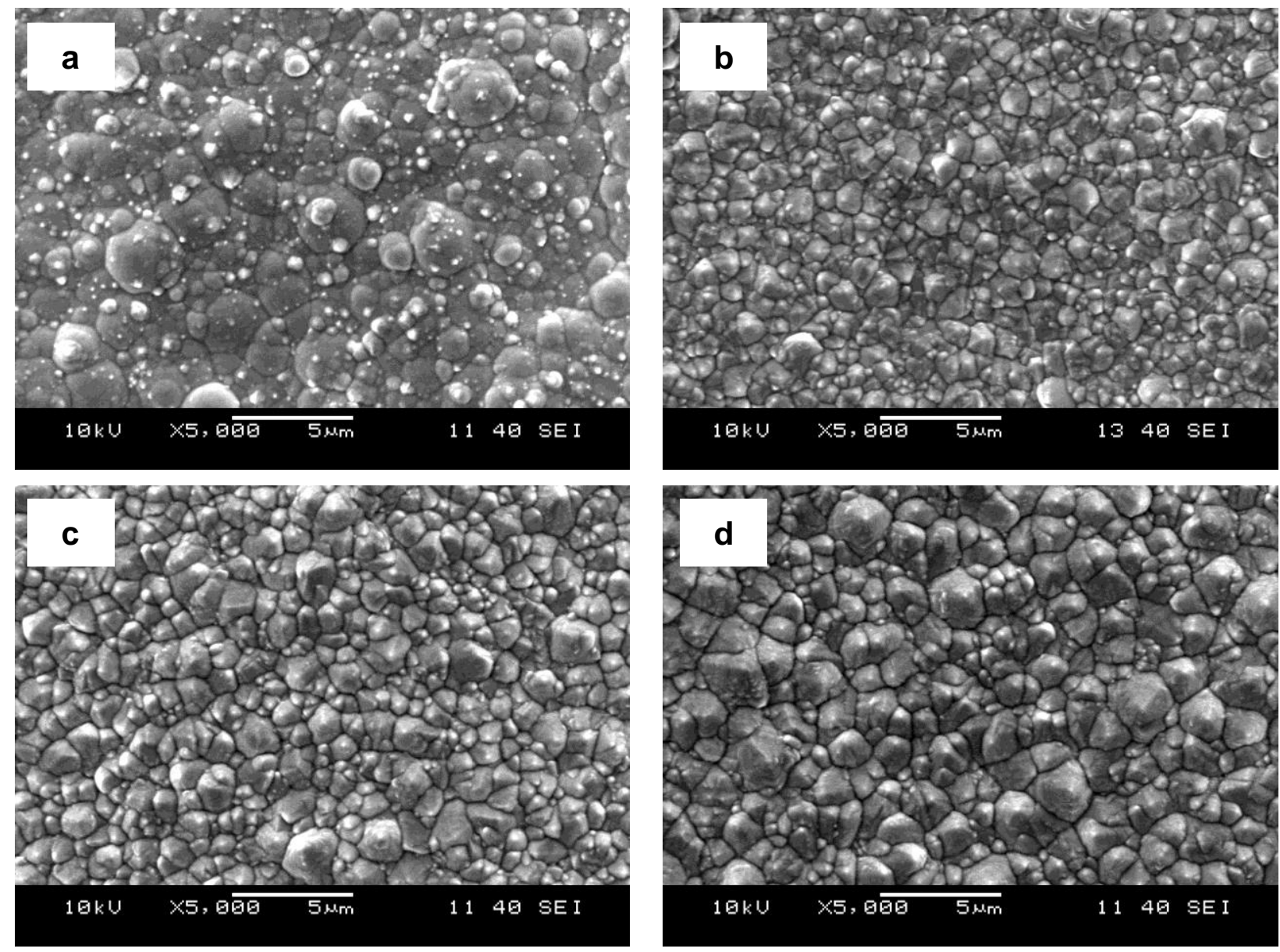

Şekil 3. Ni-Co alaşımlı kaplamaların SEM görüntüleri (a) NiCo-5 (b) NiCo-10 (c) NiCo-20 ve (d) $\mathrm{NiCo}-40$

Sonuç olarak, Ni-Co alaşımlı kaplamaların yüzey morfolojisi büyük oranda alaşım kompozisyonu ile alakalıdır. Mikroyapıdaki değişimler, çekirdeklenme ve üzerinde kobalt ve nikel hidroksitlerinin etkisi ile ilişkilidir (Tury vd., 2006).

Ni-Co alaşımlarının EDS analizi sonuçları Tablo 3'de verilmiştir.

Tablo 3. Ni-Co alaşımlı kaplamaların EDS sonuçları ve kaplama kalınlıkları

\begin{tabular}{lllll}
\hline \multirow{2}{*}{ Numuneler } & \multicolumn{2}{l}{ EDS sonuçları } & Kalınlık \\
\cline { 2 - 4 } & $\% \mathrm{Ni}$ & $\% \mathrm{Co}$ & $\begin{array}{l}\% \\
\text { Diğer }\end{array}$ & $(\mu \mathrm{m})$ \\
\hline NiCo-5 & 39.66 & 59.08 & 1.25 & $\begin{array}{l}8.1 \\
\pm 1.3\end{array}$ \\
\hline NiCo-10 & 15.56 & 82.49 & 1.95 & $\begin{array}{l}11.9 \\
\pm 1.3\end{array}$ \\
\hline NiCo-20 & 9.71 & 89.72 & 0.56 & $\begin{array}{l}9.3 \\
\pm 1.6\end{array}$ \\
\hline NiCo-40 & 7.27 & 91.24 & 1.48 & $\begin{array}{l}12.5 \\
\pm 3.8\end{array}$ \\
\hline
\end{tabular}

Ni-Co alaşımlı kaplama elektrolitinde Co miktarının artışı ile birlikte, kaplama tabakasındaki $\%$ Co miktarında da artış gözlemlenmiştir. \% Co artışı birlikte Ni-Co alaşımının \% Ni oranında azalma tespit edilmiştir. Kaplamada ki kobaltın kütlesel oranı daima elektrolit içindeki $\mathrm{Co}^{+2}$ iyonlarının konsantrasyonundan daha fazladır. $\mathrm{Bu}$ durum daha önceki araştırmacıların sonuçları ile uyumlu olan Ni-Co alaşımlı kaplamanın sıra dışı davranışı ile ilgilidir (Wang vd., 2005; Srivastava vd., 2006). Bu sıra dışı davranışı açıklayan çeşitli gerekçeler vardır. Bunlar; metal iyonları ile metal hidroksitlerinin adsorpsiyonu arasındaki yarış, metal hidroksitin oluşumu ve kaplama yüzeyinin yakınındaki pH değerindeki değişimler olarak ifade edilebilir [43, 43]. Ni-Co alaşımlı kaplama tabakasının kimyasal kompozisyonu ve yüzey morfolojisi sabit akım yoğunluğu ve $\mathrm{pH}$ eşliğinde değişken elektrolit konsantrasyonu ile farklılık göstermektedir.

Ni-Co alaşımlı kaplama tabakalarının kalınlıklarına dair yapılan ölçümlerin sonuçları 
Tablo 3'de verilmiştir. Genel olarak ortalama kaplama kalınlığı $\sim 10 \mu \mathrm{m}$ olarak tespit edilmiştir. Tabi bu kalınlığa etki eden faktörlerin başında akım yoğunluğu ve kaplama süresi gelmektedir. Artan akım yoğunluğu ve kaplama süresi ile kalınlık değeri artırılabilir. Endüstriyel kaplamalarda genel olarak elektrolitik kaplama kalınlıkları 8-12 $\mu \mathrm{m}$ arasında değişmektedir.

\section{Sonuç}

Sonuç olarak, elektrolitik kaplama metodu ile farklı Co konsantrasyonuna sahip banyolarda çelik malzeme yüzeyleri Ni-Co alaşımı ile kaplanmıştır. Co miktarındaki artışa paralel olarak alaşımlı kaplamanın mikroyapısında değişimler gözlemlenmiştir. Artan Co oranı ile kaplama tabakasının kristal yapısındaki değişim yüzey morfolojisinde de kendini göstermiştir. Yüksek oranda Co oranı daha granüler bir yapının oluşmasına neden olmuştur. Aynı zamanda alaşım tabakasındaki Co miktarıda elektrolit içinde ki Co artışı ile \%90'ı aşmıştır. Elektrolit kompozisyonu $\mathrm{Ni}-\mathrm{Co}$ alaşımında yüzey özelliklerini doğrudan etkilemektedir.

\section{Kaynaklar}

Atuanya, C.U. ve Ekweghiariri, D.I., 2017. Experimental correlation between varying processing properties and wear behaviour of ternary $\mathrm{Ni}-\mathrm{Co}-\mathrm{SiO}_{2}$ composites coating of mild steel, The International Journal of Advanced Manufacturing Technology, 88 (9-12), 25812588.

Bai, A. ve Hu, C.C., 2002. Effects of electroplating variables on the composition and morphology of nickel-cobalt deposits plated through means of cyclic voltammetry, Electrochimica acta, 47 (21), 3447-3456.

Bakhit, B. ve Akbari, A., 2013. Nanocrystalline Ni-Co alloy coatings: electrodeposition using horizontal electrodes and corrosion resistance, Journal of Coatings Technology and Research, 10 (2), 285-295.

Brenner, A., 1963. Electrodeposition of Alloys: Principles and Practice: General survey, principles, and alloys of copper and silver (Vol. 1). Academic Press.

Chen, L., Wang, L., Zeng, Z. ve Xu, T., 2006. Influence of pulse frequency on the microstructure and wear resistance of electrodeposited $\mathrm{Ni}-\mathrm{Al}_{2} \mathrm{O}_{3}$ composite coatings. Surface and Coatings Technology, 201 (3-4), 599-605.
Golodnitsky, D., Rosenberg, Y. ve Ulus, A., 2002. The role of anion additives in the electrodeposition of nickel-cobalt alloys from sulfamate electrolyte, Electrochimica Acta, 47 (17), 2707 2714.

Gomez, E., Pane, S., Alcobe, X. ve Valles, E. 2006. Influence of a cationic surfactant in the properties of cobalt-nickel electrodeposits. Electrochimica acta, 51 (26), 5703-5709.

Hassani, S., Raeissi, K. ve Golozar, M.A., 2008. Effects of saccharin on the electrodeposition of $\mathrm{Ni}-\mathrm{Co}$ nanocrystalline coatings. Journal of Applied Electrochemistry, 38 (5), 689-694.

Ishikawa, M. Enomoto, H. Matsuoka, M. ve C. Iwakura, 1994. Effect of tetraborate ions on electrodeposition of nickel-copper alloy from a pyrophosphate bath, Electrochimica Acta 39 (14), 2153-2157.

Landolt, D., 2002. Electrodeposition science and technology in the last quarter of the twentieth century, Journal of The Electrochemical Society, 149 (3), 9-20.

Lupi, C. ve Pilone, D., 2001. Electrodeposition of nickel- cobalt alloys: the effect of process parameters on energy consumption. Minerals Engineering, 14 (11), 1403-1410.

Lupi, C., Dell'Era, A., Pasquali, M. ve Imperatori, P., 2011. Composition, morphology, structural aspects and electrochemical properties of $\mathrm{Ni}-\mathrm{Co}$ alloy coatings, Surface and Coatings Technology, 205 (23-24), 5394-5399.

Ma, C., Wang, S.C. ve Walsh, F.C., 2015. Electrodeposition of nanocrystalline nickelcobalt binary alloy coatings: a review, Transactions of the IMF, 93 (2), 104-112.

Oriňáková, R., Turoňová, A., Kladeková, D., Gálová, M. ve Smith, R.M., 2006. Recent developments in the electrodeposition of nickel and some nickel-based alloys, Journal of Applied Electrochemistry, 36 (9), 957-972.

Qiao, G., Jing, T., Wang, N., Gao, Y., Zhao, X., Zhou, J. ve Wang, W., 2006. Effect of current density on microstructure and properties of bulk nanocrystalline $\mathrm{Ni}-\mathrm{Co}$ alloys prepared by JED, Journal of The Electrochemical Society, 153 (5), C305-C308.

Srivastava, M., Selvi, V.E., Grips, V.W. ve Rajam, K.S., 2006. Corrosion resistance and microstructure of electrodeposited nickel-cobalt alloy coatings, Surface and Coatings Technology, 201 (6), 3051-3060. 
Tury, B., Lakatos-Varsányi, M. ve Roy, S. 2006. Ni-Co alloys plated by pulse currents. Surface and Coatings Technology, 200 (24), 6713-6717.

Wang, L., Gao, Y., Xue, Q., Liu, H. ve Xu, T., 2005. Microstructure and tribological properties of electrodeposited Ni-Co alloy deposits. Applied Surface Science, 242 (3-4), 326-332.
Yang, Y.Y. ve Deng, B., 2011. Preparation of Ni-Co alloy foils by electrodeposition. Advances in Chemical engineering and science, 1 (02), 27.

Zhuang, Y. ve Podlaha, E.J., 2000. NiCoFe ternary alloy deposition: I. an experimental kinetic study, Journal of the Electrochemical Society, 147 (6), 2231-2236. 